\title{
A Comparison of Recreational Water Illness Awareness and Frequency of Swimming
}

\author{
Elden Chan ${ }^{1}$, Martin MacLeod ${ }^{2}$
}

${ }^{1}$ Lead Author, B. Tech Student, School of Health Sciences, British Columbia Institute of Technology, 3700

Willingdon Ave, Burnaby, BC V5G 3H2

${ }^{2}$ Supervisor, School of Health Sciences, British Columbia Institute of Technology, 3700 Willingdon Ave, Burnaby, BC V5G 3H2

\section{Abstract:}

Objective: Swimming pools have potential to create health hazards that range from physical, chemical to biological. Biological concerns center on communicable diseases which can be spread through poor hygiene practices. To prevent the spread of disease, swimmers must be informed and practice appropriate hygiene. This study aims to determine whether the frequency of swimming had an influence on swimming pool hygiene knowledge.

Method: Survey questions focusing on risk factors for communicable disease spread, and personal hygiene practices were developed using the pool operation handbooks, input from environmental health instructors at the British Columbia Institute of Technology, as well as past surveys from other studies. Pool patrons were surveyed at Hillcrest Community Centre in Vancouver. Scores from these tests were then analyzed in NCSS9 using one-way ANOVA tests and Two-Sample t-tests.

Results: 167 validated surveys were analyzed. The most often incorrectly answered questions were in regards to the duration of avoiding swimming after diarrheal illness, pre-swim hygiene, and after bathroom use hygiene. No statistically significant difference in mean test score was found in individuals that swam more than once a week, more than once a month, or less than once a month. Statistically significant differences in mean test scores were found for individuals that read signs, and age group.

Conclusion: From the data, it is observed that those who swam more frequently were not more informed on pool hygiene issues than those who swam less. The sample size $(\mathrm{n}=167)$ for this conclusion may be too small, since beta value was $87.5 \%$.

Keywords: Swimming, pool, hygiene, knowledge, survey.

\section{Introduction}

Swimming pools are facilities often associated with exercise, fitness, and betterment of health. Despite their simple appearance, they can also be associated with physical, chemical and biological hazards. Physical hazards include the potential for drowning of patrons due to lack of water clarity or pool features that can trap patrons underwater (e.g. water drain vents). Chemical hazards are found in the pool environment due to the chemicals used in treatment of the water (e.g. chlorine gas). Biological hazards refer to recreational water illnesses (RWIs), consisting of human pathogens, illnesses and diseases. While chemical and physical hazards rely on pool operator diligence in maintenance and safety, biological threats from RWIs can extend beyond a pool operator's control, and rely on pool patrons to self-regulate their own behaviours.
RWIs are communicable diseases and illnesses contracted from environments such as swimming pools, hot tubs, water parks, beaches, lakes, and in general, any body of water used for recreation (McClain, J., Bernhardt, J. M., \& Beach, 2005). Of these environments, swimming pools and hot tubs are of special concern due to their warm and moist environments, which permit pathogenic organisms to thrive (NSPF, 2012). With the number of potential pathogens and transmission methods, pool facilities pose significant public health hazards, and therefore are regulated establishments inspected by Environmental Health Officers (EHOs).

EHOs in British Columbia (BC) are mandated to inspect and ensure pool facilities are operating in adherence to provincial regulations. Inspection duties from the $\mathrm{BC}$ pool regulation focus on adequate treatment of pool water, appropriate sanitation of 
facilities and pool decks, and signage informing patrons of pool safety practices and appropriate hygiene (Pool Regulation, 2010). However, EHOs in BC lack the legislative powers to monitor, enforce, and ensure proper hygiene behaviours practiced by patrons, which are often the direct causes of RWI transmission. Thus, the EHO's role in public health protection at pool facilities may be more effective if they are involved in the development of health promotion programs and education material for the general public.

Pool operators are also responsible for ensuring safety of their patrons, and preventing RWI transmission. While maintenance activities may help reduce the environmental characteristics contributing to pathogen growth in a pool, operators encourage patrons to observe proper hygiene through signage. Despite the efforts of operators and EHOs, deficiencies in patron hygiene behaviour contribute to the spread of RWIs (Centers for Disease Control and Prevention [CDC], 2011b). Therefore, the modification and improvement of swimmer hygiene behaviour is of particular interest to protect public health.

\section{Literature Review}

\section{Factors Contributing to Pool Illnesses}

Swimmer hygiene practices are contributors to the contracting or spreading of RWI. In order to determine the best means of prevention, operators and EHOs must first understand the organisms and pathogens involved, and second, the specific behaviours that contribute to or reduce the spread of RWIs and swimmer infections.

\section{RWI Pathogens of Poor Hygiene and Illness}

Pathogenic organisms that contribute to RWIs include bacteria, viruses, fungi and parasites that may be present and transmitted through pool decks and toys, patrons' towels, goggles, and bodies, and pool water itself (National Swimming Pool Foundation [NSPF], 2012). RWIs that manifest as gastrointestinal and nongastrointestinal illnesses are often brought into the pool environment via poor hygiene or illness. The following overview is not an exhaustive list but a sampling of common illnesses and infectious agents in the swimming pool setting.

Gastrointestinal illness pathogens can come from different organisms: protozoan parasites, bacteria, and viruses. These pathogens are spread fecal-orally, meaning infected individuals or carriers of the disease release fecal material into the pool either through an accidental fecal release, or swimming while still infected and lacking proper hygiene.

Protozoans of particular concern are Cryptosporidium sp. and Giardia lamblia (NSPF, 2012; World Health Organization [WHO], 2006). Compared to other RWI pathogens, protozoans are more resistant to chemical treatment at pools, have long periods of time where an infected person can spread the disease, and require the ingestion of extremely few pathogens to become infected (NSPF, 2012). Cryptosporidium $s p$. and Giardia sp. cause diarrheal illnesses that resolve on their own, except in immunocompromised individuals (e.g. HIV/AIDS status), who can have lifelong illnesses (Health Canada, 2012a)

Bacteria of interest include Escherichia coli O157:H7 and Shigella sp. Both of which are responsible for serious infections that can lead to bloody diarrhea, and complications such as kidney failure (hemolytic uremic syndrome) and death, specifically in the case of E. coli O157:H7 (Heymann, 2008; NSPF, 2012; WHO, 2006).

Common viruses recognized as RWIs include noroviruses and rotaviruses. Noroviruses often cause vomiting and diarrhea, with symptoms suddenly appearing within 12 hours to 4 days, resolving without treatment (NSPF, 2012). Rotaviruses often cause symptomless infections in healthy adults, but may result in diarrhea that is life-threatening due to severe dehydration in young children (Health Canada, 2012b).

Non-gastrointestinal illnesses include skin, ear, and eye infections from pathogens that may be carried into the pool by infected individuals or through poor hygiene. Skin and ear infections can arise from contact with Pseudomonas aeruginosa a bacterium that is found everywhere in the environment, but are brought into the pool by individuals infected or carrying the bacterium (NSPF, 2012). Pseudomonas aeruginosa thrives in warm environments like hot tubs (NSPF, 2012). Skin infections by $P$. aeruginosa present with itchy red pustules, but generally resolve on their own without treatment (NSPF, 2012). Ear infections caused by $P$. aeruginosa result in painful ear infections, which incurs health care costs of up to $\$ 500$ million each year in the US (CDC, 2011a). Staphylococcus aureus is another bacterium that can cause skin infections, with one strain in particular of concern: Methicillin resistant S. aureus, an antibiotic resistant bacterium (NSPF, 2012). $S$. aureus contributes to skin, eye and wound infections through contact with an infected person or carrier 
(NSPF, 2012). Other skin infections include plantar warts, which is caused by a papillomavirus, and athlete's foot, which is caused by a fungus (NSPF, 2012). Both of these conditions result from infectious skin fragments left behind by infected individuals. Eye infections (conjunctivitis or pink eye) can be caused by adenoviruses, and is spread through contact with highly contagious pus discharged from the eyes of an infected person (Pond, 2005). Whether it is gastrointestinal or non-gastrointestinal, a RWI pathogen's spread in a pool is dependent on swimmer behaviours, thus modifying and controlling swimmer behaviours is paramount to prevention.

\section{Behaviours that Contribute to Illnesses}

The CDC (2011b) states that, "modifying swimmer behavior is a critical component of reducing recreational water-associated outbreaks.” To determine whether swimmer hygiene behaviour was an issue for pool hygiene, a study on indicator microbes in pool filters for poor hygiene was conducted. It was revealed that $75 \%$ of the tested swimming pools carried at least one of two indicator organisms in their filter backwash water (CDC, 2013c). The results indicated contamination of pool water by swimmers, and that there is a need for improved swimmer hygiene prior to swimming; namely, showering prior to entering a pool, and avoiding swimming during diarrheal episodes (CDC, 2013c; WHO, 2006).

Swimmers behave in ways that can decrease their risk of becoming infected or spreading infections to others. Pre-swimming behaviour includes avoidance of swimming during illness or at least two weeks after diarrheal episodes (NSPF, 2012; WHO, 2006). These actions will help prevent fecal spread diseases such as E. coli O157:H7 and Cryptosporidium sp. (NSPF, 2012; WHO, 2006). Showering thoroughly with soap focusing on cleansing the buttocks will reduce the amount of remaining fecal matter which may carry pathogens (NSPF, 2012; WHO, 2006).

During their stay visit to a pool, swimmers should avoid swallowing water, limiting the risk of ingesting fecal-orally transmitted diseases. Children should be helped and taught to minimize their intake of pool water for the same reason. Ear plugs and swim caps can help keep water out of ears and prevent infection (CDC, 2011a). Parents should take their children to the washroom or check their diapers every sixty minutes, ensuring their hands are washed, and showering prior to re-entry (CDC, 2013a; NSPF, 2012). Swimmers should not share personal items such as towels and swim goggles, to prevent the spread of $S$. aureus and adenovirus infections (NSPF, 2012). By neglecting proper hygiene and avoidance practices, RWI's may be transmitted, and the effect of poor hygiene may be observed in RWI trends.

\section{RWI Trends and Statistics}

The CDC reviewed and analyzed disease surveillance data from 2005 to 2008 for recreational water illness outbreaks. They determined that 134 outbreaks of RWI occurred from 2007 to 2008 (resulting in 13,966 cases of illness), compared to only 78 outbreaks from 2005 to 2006 (CDC, 2011b). The increase of $71.8 \%$ may be a growing trend for Cryptosporidium, but also could be due to improved surveillance methods (CDC, 2011b). Of the 134 outbreaks, 116 outbreaks occurred at facilities with treated water such as swimming pools (CDC, 2011b), illustrating a need to go beyond water treatment for prevention of RWIs.

In the 2007 report on BC's recreational water illnesses, it is stated that evidence attributing disease to recreational waters in BC is lacking, and that improvements are required in monitoring and surveillance efforts (BC Ministry of Health, 2007).

The BC Ministry of Health states that, in comparison with American statistics from the CDC, and the increasing trend of RWIs in the US, the estimates made for BC's trend is low enough to be a low priority regarding public health concerns (BC Ministry of Health, 2007). Despite these low trends and lack of priority, infections from Cryptosporidium sp., Giardia lamblia and Shigella sp. are still prevalent in BC, providing potential for a RWI outbreak.

The BCCDC (British Columbia Centre for Disease Control) 2012 annual report described waterborne diseases without distinction for drinking or recreational waters. The report states that cryptosporidiosis cases have risen in 2012, to 1.6 from 1.2 cases per 100,000 population, while shigellosis and giardiasis were stable at 3.8 and 13.3 cases per 100,000 population, respectively (BCCDC, 2013). By comparison Vancouver specifically showed rates of 1.6 per 100,000 population for cryptosporidiosis, 9.8 per 100,000 population for shigellosis, and 19.4 per 100,000 population for giardiasis (BCCDC, 2013). From these statistics, it is observed that BC and Vancouver both have endemic potential RWI outbreak pathogens. All 
that is needed for a RWI outbreak is an opportunity for infected individuals or carriers to bring these pathogens into the pool environment; therefore, measures put towards educating swimmers are necessary.

\section{Implications for Swimming Pools}

\section{Signage Requirements}

In $\mathrm{BC}$, section 8 of the pool regulation states that operators must post a sign with specific statements that relate to prohibiting behaviours that can lead to injury, and lack of personal hygiene (Pool Regulation, 2010). In terms of communicable diseases, the regulation states operators must post signage that prohibits entering the pool while ill with open sores, bandages, discharging eyes and ears, or failing to shower first ( $\mathrm{Pool}$ Regulation, 2010). Unfortunately section 8 does not make a specific mention of diarrhea, but rather a vague reference to illness, when guidelines recommend avoidance of swimming specifically with diarrheal illnesses (CDC, 2013a, 2013c; WHO, 2006). Where healthy hygiene behaviours fail, treatment of pool water remains the last resort to prevent RWI spread.

\section{Treatment Requirements}

EHOs routinely inspect pool facilities and focus on treatment methods and measuring disinfectant levels with test kits. While the pool regulations in BC prescribe minimum concentrations of disinfectants in pool water (Pool Regulation, 2010), pathogens such as adenovirus, Cryptosporidium sp., and Giardia lamblia are highly resistant to chlorine and bromine treatment (Health Canada, 2012a; NSPF, 2012; Victoria Department of Health, n.d.). For example, Cryptosporidium $s p$. at free available chlorine levels of $1.0 \mathrm{ppm}$ would require 255 hours for deactivation of the pathogen (NSPF, 2012), an impractical timeframe when the threat after an accidental fecal release or poor preswimming showering is immediate. For example a person releases 6,000,000 Staphylococcus aureus bacteria within 15 minutes of immersion in water (CDC, 2013b)

Other disinfection methods exist, such as ozone and UV, which are effective against inactivating Cryptosporidium sp. and Giardia lamblia (Health Canada, 2012a), but have no regulatory requirements or standards to meet in BC. Unless regulations are changed and require all pool facilities to install ozone or UV treatment processes, the threat of chlorine resistant pathogens remain, and the practical means of prevention is in education and improvement of swimmer's hygiene behaviours.

\section{Necessity for Health Promotion}

In order to effect change in pool hygiene behaviours, vulnerability and severity are factors that must be made aware in the swimmer population (McClain, J., Bernhardt, J. M., \& Beach, 2005). Educational and health promotion campaigns can bring awareness and enable swimmers to adopt RWI preventative behaviours.

\section{Educational Campaigns}

A recent campaign against poor pool hygiene behaviour was the Utah "No Crypto" campaign, which was launched during the summer following a large outbreak of cryptosporidiosis in 2007 (CDC, 2012). The campaign focused on engaging the Utah population's interest through radio, television, posters at swimming pools, and a website, while disseminating information on healthy hygienic practices. Since implementation of the program, Utah has not observed RWI outbreaks between 2008 and 2011 (CDC, 2012). As a result, the campaign may be an effective example of increasing knowledge to prevent the spread of RWIs.

\section{RWI Knowledge Surveys}

To determine whether Utah residents had an increased awareness or knowledge of RWIs, a survey was sent out in 2008 at the beginning of the "No Crypto" campaign, and a year after the campaign in 2009. The results in 2009 were then compared to national responses. From 2008 to 2009, the correct response of avoiding swimming while having diarrheal illness increased from $96.1 \%$ to $100 \%$ in Utah residents, which is higher than 2009 results from residents of other states at 78.4\% (CDC, 2012). To further illustrate the efficacy of the educational campaign, the 2009 survey also asked questions of the necessity of showering or bathing with soap prior to swimming, and whether or not the participant had heard of RWIs. $76.6 \%$ of Utah respondents and $19.1 \%$ of respondents in other states had heard of RWIs (CDC, 2012). 80.3\% of Utah respondents answered correctly, whereas 53\% of respondents in other states were correct for showering practices (CDC, 2012).

The results of improved RWI knowledge and absence of RWI outbreaks in Utah, illustrate the success and efficacy of health promoting campaigns. To reduce the risk of RWI spread and cryptosporidiosis in particular, the authors state that by reminding swimmers 
of the vulnerability and severity of RWIs, and educating them on preventative practices, RWI health promotion campaigns may increase confidence and enable swimmers to adopt healthy pool hygiene behaviours.

\section{Frequency of Exposure to Hygiene}

Research on swimmer hygiene knowledge is lacking, however, other studies show that frequent prompt can help improve or maintain healthy behaviours, and reduce unhealthy ones in terms of weight loss (Fry \& Neff, 2009). Prior to entering swimming pools, swimmers are presented with pool rules signs that remind them of safe practices.

Therefore, it may be possible that swimmers frequently exposed to the pool rules may recall proper hygiene protocols and retain knowledge better than those who infrequently visit the pool.

\section{Spray Park Hygiene Knowledge}

Past research exists for spray parks, which have similar routes of transmission of communicable diseases (i.e. through water) and similar pathogens with swimming pools. Through questionnaires, it was determined that spray park attendees showed a lack of understanding in terms of health risks associated with spray parks leading to poor hygiene behaviour and risk of disease transmission (Nett, R.J, Toblin R., Sheehan, A., Huang, W., Baughman A., Carter, 2010). In the context of swimming pools, this lack of knowledge of health risks with recreational waters in general may lead to poor hygiene behaviour

\section{Purpose of Research}

While pool operators and EHOs can work together to ensure facilities meet regulatory requirements, minimize risks of pools that harbour pathogens, and provide a safe environment for swimmers to enjoy their pool facility visits, pool patron behaviours are ultimately responsible for the introduction of RWIs. Due to the lack of research in assessing swimmer hygiene knowledge, the purpose of this study was to determine the level of knowledge of pool patrons in RWI prevention, and whether their frequency of swimming affects their knowledge. This was done through surveying and comparing hygiene knowledge test scores from swimmers. The results may assist in determining whether or not RWI health promotion campaigns are necessary, and what areas of swimmer hygiene knowledge are in deficit and require improvement.

\section{Methods and Materials}

An in-person self-administered questionnaire at Hillcrest Community Center, a facility with a swimming pool, fitness centre, and ice rink. The survey consisted of 5 demographics questions, 2 validation questions, and a knowledge quiz with 10 RWI related questions. A table was set up outside of the fitness centre and swimming pool change rooms to greet visitors, introduce the survey, explain cover letter and consent material, and provide them with a copy of the survey. Upon completion of the survey, participants had the option of filling out a form for an incentive prize draw. An answer key was used to compare and score participant responses, and ensure consistency across responses.

\section{Inclusion and Exclusion Criteria}

Since the survey was administered in-person, any guests of the community centre were eligible to participate. Guests under the age of 19 were excluded as this was the age of majority in BC, and avoided complications of requiring parental consent (Fraser Health, 2009). Additionally, pool staff such as life guards and pool maintenance staff were excluded as they may have had specialized training in RWI prevention and education.

\section{Reliability and Validity}

Reliability is defined as consistent measurement between each participant, and to what extent the study is repeatable (Heacock \& Sidhu, 2013; Office of the Auditor General of Canada, 2007). The use of a standardized survey is inherently high in reliability, since variability in administration of the survey was minimal, the questions were the same for each participant as well as the answers, and instructions were provided to ensure participants respond appropriately to questions. Questions in this study were close ended with specific answers, allowing for reliable responses by limiting variability in interpretations by participants, and the investigator when reviewing submissions. An option to select "Don't know" as a response was intended to prevent guessing, and was treated as incorrect answers. This way, knowledge and understanding of RWIs can more accurately be gauged. Validity is a method's ability to accurately measure or estimate what the research question intends to find out. (Heacock \& Sidhu, 2013; Office of the 
Auditor General of Canada, 2007). Validity exists in two forms, internal and external.

Internal validity is how accurately or appropriately the method and collected data can draw conclusions relevant to the research question (Heacock \& Sidhu, 2013). In order to draw relevant conclusions to the research question of RWI knowledge and frequency of swimming, the population surveyed must have swimmers. Carrying out the survey at the community centre assists with this, as swimmers were more likely to frequent these locations compared to other public spaces. Threats to internal validity in this study were participants who have specialized knowledge of RWI control, for example swimming pool operators or lifeguards. If pool staff participate, they may inappropriately skew survey results, leading to a higher average score in their respective demographic group. To control for these threats to internal validity, a disclaimer which excludes pool staff and operators was part of the cover letter, and a validation question was included in the survey to check for their participation.

Another contribution to internal validity is how well the questions of the survey measure participant knowledge. The knowledge questions in the survey were generated from reference materials (NSPF, 2012), previous surveys (CDC, 2012), and discussions with a pool hygiene instructor (M. MacLeod, personal communication, November 8, 2013) to ensure questions were relevant and address RWIs appropriately.

External validity is how well the results of the study can be generalized to the larger population (Heacock \& Sidhu, 2013; Myers, 2006). The population tested in this study included visitors of Hillcrest Community Centre. These participants may differ from the general public as they may have behaviours that were more proactive in healthy living, such as exercise, personal hygiene, and an interest in seeking out health topics. Therefore, the external validity of this study may be limited to community centre guests, and their knowledge of pool hygiene and RWIs.

\section{Ethical Considerations}

Since this study is conducted on human participants, ethical concerns of beneficence and autonomy were considered (Heacock \& Sidhu, 2013). The paper survey does not pose harm or discomfort to the participant, and provides the benefit of contributing to scientific knowledge, which was sufficient justification for beneficence (Heacock \& Sidhu, 2013).
The survey method complies with autonomy requirements as participants were fully informed on the purpose of the study, and the data that was collected, and ensured of confidentiality with regards to their responses. Informed consent was obtained through signing of the cover letter. Furthermore, participants were free to choose whether or not they wish to participate, as well as able to refuse completion of the survey should they become uncomfortable for any reason.

\section{Pilot Studies}

5 volunteers were given a copy of the cover letter and the survey. Volunteers provided feedback on clarity of instructions, informed consent, and identify leading, confusing, or unclear questions, as well as any discomfort in answering questions. Volunteers were timed to determine the time required to complete the survey. Adjustments to wording, number of questions were made to improve clarity and a completion time of 1-2 minutes.

\section{Statistical Analyses}

\section{Description of Data}

The data collected in this study was both categorical and numerical. Categorical data were separated into ordinal and nominal data, each comprise of mutually exclusive datum, with the former having a sequential or ordered nature (Statistics Canada, 2013). Ordinal and nominal data consisted of demographic information categorized into age, gender, frequency of swimming, and education level. Numeric data consisted of test scores of the participants. These test scores represent discrete numerical data which represent finite numbers (i.e. questions can either be awarded 1 or 0 points, nothing in between) (Statistics Canada, 2013).

\section{Descriptive Statistics}

Demographic data collected was used to generate charts to illustrate the population surveyed.

Additionally, test scores was analyzed for mean, mode, median, ranges, and standard deviations of test scores of participants. A ranked list of fewest to most correctly answered questions was also created.

\section{Inferential Statistics}

To determine whether mean test scores were statistically different between demographic groups, the two-sample t-test and one-way ANOVA test were used (Heacock \& Sidhu, 2013; Moore \& McCabe, 2006). Test scores that were matched with demographic data 
with only 2 responses were analyzed using the twosample t-test, while other demographic questions with more than two responses, and were analyzed with the one-way ANOVA test. Post-hoc testing was carried out on statistically significantly different mean values in a group using the Tukey-Kramer test. Both t-tests and one-way ANOVA were carried out at a p-value $=0.05$. Groups that contained too few responses would be combined in broader categories.

\section{Software Packages}

Descriptive statistics were processed, analyzed and presented using Microsoft Excel 2010 (Microsoft, 2010). Inferential statistics were analyzed using NCSS 9 (Hintze, 2013).

\section{Results}

\section{Response and Data Collection}

A total of 200 surveys were issued, and 167 surveys were valid and analyzed. 7 were lost to nonreturn, and 26 failed validation criteria. Tabulated responses and excluded data can be retrieved from http://goo.gl/KK9bdo.

\section{Descriptive Statistics}

Demographic data of participants are summarized in Figure 1. There were insufficient responses for age groups 65+, no-post secondary education, and swimming less than once a year and non-swimmers. Overall, participants on average correctly answered 6 out of the 10 questions (mean, mode and median of 6) with a standard deviation of 1.64. A ranked list from fewest to most correct responses can be found in Table 1. The questions that focused on prevention of fecal spread diseases were most frequently answered incorrectly. Avoidance of swimming post diarrheal symptoms had the fewest correct responses, which only $6 \%$ of participants answered correctly.

\section{Inferential Statistics}

Swimming frequency, education and gender did not show a statistically significant difference in mean test scores, while age and reading of signs did, as summarized in Table 2. Therefore, the null hypothesis is not rejected, and there was no observed difference in mean score value between people who swam more frequently than those who swam less. Power was low and beta was high for statistically significant and nonsignificant, indicating a sample size of 167 may be too small at risk of errors.

Figure 1. Summary of Surveyed Population

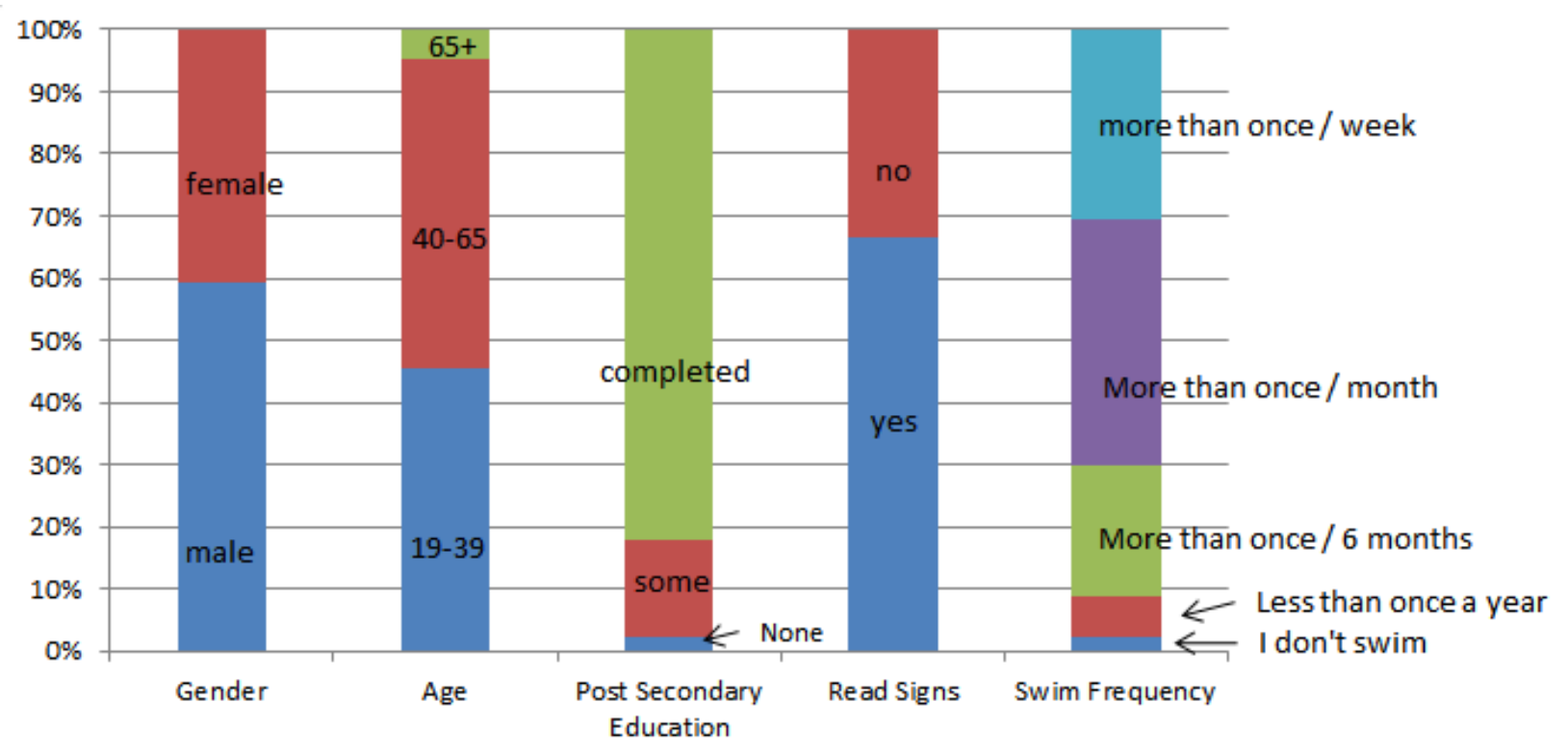


Table 1. Questions ranked from least to most correctly answered

\begin{tabular}{|r|l|c|c|}
\hline Q\# & Content & \# correct & $\begin{array}{l}\% \\
\text { Correct }\end{array}$ \\
\hline 8 & How long to wait after having diarrhea, before swimming & 10 & $6.0 \%$ \\
\hline 7 & Shower with soap after using the bathroom, before returning to pool & 52 & $31.1 \%$ \\
\hline 9 & Showering with soap before entering pool & 52 & $31.1 \%$ \\
\hline 2 & Cl kills germs instantly & 97 & $58.1 \%$ \\
\hline 3 & Swim with cuts or rashes & 103 & $61.7 \%$ \\
\hline 10 & Types of infections from swimming pools & 110 & $65.9 \%$ \\
\hline 6 & Urination affects pool water disinfection & 124 & $74.3 \%$ \\
\hline 4 & pool water = drinking water & 152 & $91.0 \%$ \\
\hline 5 & shower after swim/hot tub & 154 & $92.2 \%$ \\
\hline 1 & poor pool water maintenance causes illness & 157 & $94.0 \%$ \\
\hline
\end{tabular}

Table 2. Summary of Inferential Statistics

\begin{tabular}{l|l|l|r|r|r|}
\hline Demographic & Type of Test & Ho $^{*}$ & p-value & Power & Beta \\
\hline Education & Two Sample T & Cannot reject & 0.18 & 0.25 & 0.75 \\
\hline Age & Two Sample T & Reject & 0.0065 & 0.659 & \\
\hline Swimming Frequency & One Way Anova & Cannot reject & 0.44 & 0.125 & 0.875 \\
\hline Gender & Two Sample T & Cannot reject & 0.2 & 0.258 & 0.742 \\
\hline Reading signs & Two Sample T & Reject & 0.0036 & 0.745 & \\
\hline p-value and power at $\alpha=0.05$ & & & \\
*Ho = the demographic does not show a difference in mean scores & & \\
* \\
*Ha = the demographic shows a difference in mean scores
\end{tabular}

\section{Discussion}

While Fry \& Neff showed improved and retained healthy behaviours through health promotion and prompting (2009), the present study was unable to show a difference in mean scores between frequent and less frequent swimmers. In combination with low average knowledge scores, these results indicate that frequent swimmers do not have better or worse knowledge than less frequent swimmers, and that this knowledge is very limited. Poor swimming pool hygiene knowledge may limit swimmers abilities to practice healthy behaviours resulting in RWI outbreaks (Nett, R.J, Toblin R., Sheehan, A., Huang, W., Baughman A., Carter, 2010)

Previous studies have found that perceived risk for illness improves healthy behaviours and practices (Usfar, Iswarawanti, Davelyna, \& Dillon, n.d.; Vivas et al., 2010). The present pool knowledge survey indicated that $94 \%$ of respondents believed poor pool water maintenance can cause illness, and $91 \%$ believed that pool water quality was different from drinking water quality. Despite these responses indicating swimmers perceiving a potential health risk to swimming, knowledge regarding swimming hygiene and avoidance remained low. This may indicate that swimmers perceive a potential health hazard, and increase their likelihood of seeking information or learning about RWIs and proper hygiene practices, but their scores do not reflect sufficient knowledge. A common response during the survey was that participants often did not know the answer, and were eager to find out answers afterwards. Furthermore, swimmers were often surprised by the length of avoidance after diarrheal illness. These comments show that swimmers may be overconfident in their hygiene knowledge, and did not seek out correct information. Therefore correct hygiene and RWI facts should be provided at pools to correct misconceptions, and supplement knowledge deficits.

Reading warning signs and age were shown to have statistically significant difference in test scores, while swimming frequency, gender, and education did not. These differences are reasonable, since reading the signs will directly impart some knowledge to swimmers, but becomes irrelevant if frequent swimmers ignore them. Age may relate to swimmers personal interest in hygiene (e.g. being elderly may result in swimmers being more aware of risks of severe illness due to reduced immunity). Swimming pool hygiene might not be part of a regular school curriculum, and perhaps neither gender takes greater interest in 
swimming hygiene over the other, which explains the lack of significance in these two demographic categories.

The questions with content regarding duration of avoidance after diarrheal illness, showering after using the bathroom, and showering before entering a pool, were most frequently answered incorrectly. All three of these questions lend concern for fecal contamination. Diarrheal illness may be indicative of cryptosporidiosis or giardiasis, and minute quantities of feces can contain infectious doses of pathogens or their cysts and oocysts (NSPF, 2012). If patrons lack the knowledge to stay away from swimming pools during diarrheal illness, there is a risk of disease outbreak including cryptosporidiosis and giardiasis, since they may still shed the pathogen.

A potential explanation for the lack of knowledge of avoidance times may be due to the fact that the last swimming pool outbreak of cryptosporidiosis was in 2004 in the city of Surrey (Health Canada, 2004). The present study was carried out nine years after the outbreak, and it is possible that the Metro Vancouver's residents have forgotten the outbreak, or it is no longer a perceived threat to them. Since a cryptosporidiosis outbreak is no longer a perceived threat, self-education and knowledge of avoidance times may be a lower priority for swimmers, resulting in few correct responses for question 8 of the survey. From previous studies, perceived threats can improve hygiene knowledge and behaviour (Usfar, et al., n.d.; Vivas et al., 2010), and therefore educational materials or communications must focus on increasing the perceived threat of disease outbreak when discussing avoidance times.

Knowledge scores also show that swimmers did not know to shower thoroughly with soap even after going to the bathroom, and therefore trace amounts of feces can be retained (WHO, 2006), which presents a risk for disease transmission. The lack of understanding for proper hygiene in a recreational water facility are key risk factors for outbreaks (CDC, 2012; Nett, R.J, Toblin R., Sheehan, A., Huang, W., Baughman A., Carter, 2010). An education campaign may help improve swimmer knowledge.

Utah’s “No Crypto” crypto campaign was a success in that they did not experience any other outbreaks after 2008, and citizens of Utah scored much higher than the national average in swimming hygiene knowledge scores (CDC, 2012). The present study has indicated a knowledge deficit in the swimmer population at the surveyed community centre, which may benefit from a campaign similar to "No Crypto". However, since BC has not observed swimming pool related cryptosporidiosis outbreaks since 2004 (Health Canada, 2004), there may be little incentive for funding an education campaign. Therefore, the data from the present study should serve as a warning to public health officials and pool operators that swimmers are lacking important hygiene knowledge, and conditions for outbreaks, such as presence of pathogens and ignorance of personal hygiene measures, are present.

\section{Recommendations}

A large population of swimmers could not read the English survey, and this is of particular concern since the pool rules are also in English only, which may result in high risk individuals entering pool environment. Therefore, multilingual rules and hygiene guides are necessary to educate and reduce risk of disease outbreak in non-English speaking populations.

Swimming pool hygiene knowledge was limited as indicated by the results, and therefore interventions are needed to provide swimmers with more information. Fry \& Neff found that prompting via text messages, email, periodic phone calls, and community message board reminders were highly effective in improving healthy behaviours (2009). While these methods may be too invasive and become a nuisance, prompting at a swimming pool environment can take the form of reminders from staff during payment or check-in for swimming. Additionally, community center newsletters could include RWI information with swimming pool schedules.

EHOs can become involved when advising on the revision of pool regulations, bringing up the fact that Canada's culturally diverse demographics warrants multilingual signs and rules. EHOs also work directly with pool operators during inspections, and can provide advice on key issues to focus on in signage. For example, EHOs can use the results from this survey decide what kind of information to provide operators when helping them develop signage. From the current data, EHOs may choose to focus on diarrheal illness prevention at pools. This targeted approach allows EHOs prepare for specific topics and better improve risk communications, raise awareness, and swimmers' perception of risk, to increase swimmers' knowledge and encourage proper swimming hygiene behaviour 


\section{Limitations and Improvements}

During the survey process, limitations of the survey questions and population were revealed. Many swimmers could not read English, and therefore could not participate, by missing such a large population of swimmers, this study is only applicable to the English speaking population. Furthermore, it was difficult to obtain a minimum number of responses in groups such as the elderly, or those who didn't swim, resulting in the broader ranges of ages and swimming frequency. This reduces the resolution in demographic categories, and that it is unable to show more specific differences between groups. Thus, alternate language versions (Chinese in particular), and a larger sample size can remedy the issues of reaching different groups.

The questions chosen for the survey limited the ability to determine perceived risk in certain swimmers. Many of the swimmers came as a family, and the survey questions did not target whether parents encouraged or facilitated proper hygiene for young children, or whether they took extra personal hygiene precautions to either protect or role model for their children. Additionally, at risk group status was not determined due to privacy concerns, these individuals may have special interest in personal hygiene and protection. To resolve both limitations for perception of risk, participants could be asked how safe they feel regarding pools and RWIs.

\section{Errors}

The present study has a sample size of 167 , which may have been too low due to low power and high beta values as shown in Table 1. Power values were less than 0.80 for both sign reading and age, which is a potential false rejection of the null hypothesis, a type I error. High beta values were observed for education, gender, and frequency of swimming, which means there may be a high probability that the inability to reject the null was a false negative, or type II error. In both these situations, large sample sizes are needed to increase power and decrease beta.

\section{Future Studies}

1.) Revisiting and reanalyzing data from the present study to determine if there is an association between frequency of swimming and reading the pool signs.

2.) Create a survey to determine the level of perceived risk of RWIs in swimming pools and where risk factors stem from.
3.) Repeating the survey in other languages (Chinese in particular)

4.) Create a survey focusing on aspects of signage and age and their effects on swimming hygiene knowledge.

\section{Conclusion}

From the present study, swimming frequency does not appear to affect swimmers knowledge on swimming pool hygiene, despite the expectation that frequent swimmers may be more frequently exposed to risks and pool hygiene rules and therefore more educated in the subject matter. The average knowledge test score was 6 out of 10 , with crucial questions such as swimming avoidance duration after diarrheal illness, preswimming and after bathroom use hygiene being the most often incorrectly answered. A large proportion of patrons cannot read English and represent a large population at risk for spread of RWIs.

\section{Acknowledgements}

The author would like to express his thanks to all parties involved in the production of this study. This work would not have been possible without the generosity and support of the staff at Hillcrest Community Centre, and the participants who shared their valuable time to support research. This study also would not have been possible without the support of the Environmental Health teaching staff at the British Columbia Institute of Technology. Their advice, guidance, and experience have helped shaped this project, and ensure its success. Finally, the author would like to thank the Environmental Health Class of 2012-2014, who provided advice, recommendations, and boundless encouragement.

\section{Competing Interests}

The authors declare they have no competing interests.

\section{References}

BC Ministry of Health. (2007). Core public health functions for BC: Evidence review - Water quality: Recreation water. BC Ministry of Health. Retrieved from http://www.health.gov.bc.ca/publichealth/pdf/Water_Quality_Recreational_WaterEvidence_Review.pdf

BCCDC. (2013). British Columbia annual summary of reportable diseases 2012. Vancouver. Retrieved 
from

http://www.bccdc.ca/NR/rdonlyres/F30377E3-

D33E-4755-B3F4-

6844E01BD678/0/FinalAR2012.pdf

CDC. (2011a). Estimated burden of acute otitis externa - United States, 2003-2007. MMWR: Weekly, 60(19), 605-609. Retrieved from http://www.cdc.gov/mmwr/preview/mmwrhtml/ mm6019a2.htm

CDC. (2011b). Surveillance for waterborne disease outbreaks and other health events associated with recreational water - United States, 20072008. MMWR: Surveillance Summaries, 60(12), 1-32. Retrieved from http://www.cdc.gov/mmwr/preview/mmwrhtml/s s6012a1.htm

CDC. (2012). Promotion of healthy swimming after a statewide outbreak of cryptosporidiosis associated with recreational water venues Utah, 2008-2009. MMWR: Weekly, 61(19), 348-352. Retrieved from http://www.cdc.gov/mmwr/preview/mmwrhtml/ mm6119a5.htm?s_cid=mm6119a5_e

CDC. (2013a). CDC - Hygiene - Healthy water. Retrieved October 10, 2013, from http://www.cdc.gov/healthywater/hygiene/swim ming/

CDC. (2013b). Hygiene fast facts. Retrieved from http://www.cdc.gov/healthywater/hygiene/fast_f acts.html

CDC. (2013c). Microbes in pool filter backwash as evidence of the need for improved swimmer hygiene - Metro-Atlanta, Georgia, 2012. MMWR: Weekly, 62(19), 385-388. Retrieved from

http://www.cdc.gov/mmwr/preview/mmwrhtml/ mm6219a3.htm?s_cid=mm6219a3_w

Fraser Health. (2009). FAQ: Informed consent.

Retrieved from

http://research.fraserhealth.ca/about_us/faq/infor med_consent/

Fry, J. P., \& Neff, R. A. (2009). Periodic prompts and reminders in health promotion and health behavior interventions: systematic review. Journal of Medical Internet Research, 11(2), e16. doi:10.2196/jmir.1138

Heacock, H., \& Sidhu, B. (2013). Research design \& data collection. Lecture conducted at British Columbia Insititue of Technology, Burnaby, BC.
Health Canada. (2004). An outbreak of cryptosporidium parvum in a Surrey pool with detection in pool water sampling. Canada Communicable Disease Report, 30(1), 61-65. Retrieved from: http://www.phac-aspc.gc.ca/publicat/ccdrrmtc/04pdf/cdr3007.pdf

Health Canada. (2012a). Enteric protozoa: Giardia and cryptosporidium. Retrieved from http://www.hcsc.gc.ca/ewh-semt/pubs/watereau/protozoa/index-eng.php

Health Canada. (2012b). Guidelines for Canadian recreational water quality (Third Edit.). Ottawa. Retrieved from http://www.hc-sc.gc.ca/ewhsemt/pubs/water-eau/guide_water-2012guide_eau/index-eng.php

Heymann, D. L. (Ed.). (2008). Control of communicable diseases manual (19th ed.). Washington: American Public Health Association.

Hintze, J. (2013). NCSS 9. Kaysville, Utah, USA: NCSS, LLC. Retrieved from www.ncss.com

McClain, J., Bernhardt, J. M., \& Beach, M. J. (2005). Assessing parents' perception of children's risk for recreational water illnesses. Emerging Infectious Diseases, 11(5), 670-676. Retrieved from http://wwwnc.cdc.gov/eid/article/11/5/040779 article.htm

Microsoft. (2010). Microsoft Excel.

Moore, D. S., \& McCabe, G. P. (2006). Introduction to the Practice of Statistics (5th ed.). W.H. Freeman and Company.

Myers, D. G. (2006). Psychology (8th ed.). New York: Worth Publishers.

Nett, R.J, Toblin R., Sheehan, A., Huang, W., Baughman A., Carter, K. (2010). Nonhygienic behavior, knowledge, and attitudes among interactive splash park visitors. Journal of Environmental Health, 73(4). Retrieved from http://www.cdc.gov/nceh/ehs/Docs/Nonhygienic _Behavior_Splash_Parks.pdf

NSPF. (2012). Pool \& spa operator handbook. Colorado Springs: National Swimming Pool Foundation.

Office of the Auditor General of Canada. (2007). The reliability and validity of survey information. Retrieved November 09, 2013, from http://oagbvg.gc.ca/internet/English/meth_gde_e_19726.ht $\mathrm{ml}$

Pond, K. (2005). Water recreation and disease. London: World Health Organization. Retrieved from 
http://www.who.int/water_sanitation_health/bath ing/recreadis.pdf

Pool Regulation. , Pub. L. No. B.C. Reg. 296/2010 (2010). Retrieved from http://www.bclaws.ca/EPLibraries/bclaws_new/ document/ID/freeside/296_2010

Statistics Canada. (2013). Variables. Statistics: Power from data! Retrieved from http://www.statcan.gc.ca/edu/powerpouvoir/ch8/5214817-eng.htm

Usfar, A. A., Iswarawanti, D. N., Davelyna, D., \& Dillon, D. (n.d.). Food and personal hygiene perceptions and practices among caregivers whose children have diarrhea: a qualitative study of urban mothers in Tangerang, Indonesia. Journal of Nutrition Education and Behavior, 42(1), 33-40. doi:10.1016/j.jneb.2009.03.003

Victoria Department of Health. (n.d.). Cryptosporidium and cryptosporidiosis - information for swimming pool managers: Water. Retrieved from http://www.health.vic.gov.au/water/recreational/ cryptosporidium.htm

Vivas, A. P., Gelaye, B., Aboset, N., Kumie, A., Berhane, Y., \& Williams, M. A. (2010). Knowledge, attitudes and practices (KAP) of hygiene among school children in Angolela, Ethiopia. Journal of Preventive Medicine and Hygiene, 51(2), 73-9. Retrieved from http://www.pubmedcentral.nih.gov/articlerender. fcgi?artid=3075961\&tool=pmcentrez\&rendertyp $\mathrm{e}=$ abstract

WHO. (2006). Guidelines for safe guidelines for safe recreational water environments. Volume 2: Swimming pools and similar recreational-water environments. Geneva: WHO Press. Retrieved from http://www.who.int/water_sanitation_health/bath ing/bathing2/en/index.html 